\title{
La resolution des conflits juridiques et politiques par la justice constitutionnelle de la Republique de Djibouti
}

\section{Resolution of Legal and Political Conflicts by the Constitutional Justice of the Republic of Djibouti}

\section{Mohamed ABDILLAHI BAHDON*}

RÉSUMÉ: Contrairement à un régime autoritaire, dans le régime "démocratique", le conflit fait partie du jeu politique. Il repose sur l'opposition pacifique entre les acteurs/ es de la scène sociopolitique. Cet article analyse le rôle du conseil constitutionnel de la République de Djibouti dans la résolution des conflits juridiques et politiques. La première partie est consacrée à la nécessité de la justice constitutionnelle et à son importance dans un régime non autoritaire. C'est une question importante. Dans la deuxième partie, l'évolution de la perception sur la justice dans ce pays sera analysée entre deux contextes différents 1977 et 1992. La quatrième partie porte sur les éléments de définition du conflit juridico-politique dans un régime politique où il est un fait à gérer par une institution juridictionnelle. Dans la cinquième partie, les mécanismes prévus par la constitution et les lois organiques, mécanismes traduisant un changement culturel apparent des membres de l'élite politique, seront traités. L'objet traité par la sixième partie est la compétence exclusive en matière électorale, qui constitue l'un des foyers de conflits juridiques et politiques.
ABSTRACT: Unlike an authoritarian regime, in the "democratic" regime, conflict is part of the political game. It is based on peaceful opposition between actors in the sociopolitical scene. This article analyzes the role of the Constitutional Council of the Republic of Djibouti in the resolution of legal and political conflicts. The first part is devoted to the need for constitutional justice and its importance in a non-authoritarian regime. This is an important question. In the second part, the evolution of perception on justice in this country will be analyzed between two different contexts 1977 and 1992. The fourth part deals with the elements of definition of the legal and political conflict in a political regime where it is a fact to be managed by a jurisdictional institution. In the fifth part, the mechanisms provided by the constitution and the organic laws, mechanisms translating an apparent cultural change of the members of the political elite, will be dealt with. The subject dealt with in Part Six is exclusive electoral jurisdiction, which is one of the hotbeds of legal and political conflict.

* Universidad de Murcia, España. Correo electrónico: bahdonabdillahi@gmail.com. 
Et enfin, la septième partie analysera la résolution de ces conflits par le recours aux ressources que disposent les acteurs/es et les candidates. Ce sont des voies de résolution pacifique.

Mots clés: conflit, compétence exclusive, contentieux électoral, opposition pacifique, saisines, résolution pacifique.
And finally, the seventh part will analyze the resolution of these conflicts by the recourse to the resources that the actors and the candidates have. These are ways of peaceful resolution.

Keywords: Conflict, Exclusive Jurisdiction, Electoral Disputes, Peaceful, Opposition, Referrals, Peaceful Resolution.

\begin{abstract}
SUMMARY: I. Introduction. II. Nécessité ou mode de la juridiction constitutionnelle. III. Changement relatif de sa perception dans le nouveau régime politique. IV. Les éléments constitutifs d'un conflit juridique et politique. V. Les mecanismes de la résolution des conflits juridiques et politiques. VI. La compétence exclusive en matière de contentieux électoral. VII. Les saisines du conseil constitutionnel comme moyen de resolution des conflits juridiques. VIII. Conclusion.
\end{abstract}

\title{
I. INTRODUCTION
}

L'analyse de la justice constitutionnelle en Afrique se heurterait, selon le professeur Luc Sindjoun ${ }^{1}$ à de nombreux obstacles épistémologiques. Mais ces obstacles ne peuvent décourager les débats juridiques, politiques et sociaux sur cette institution; qui est, plus que jamais, au centre de la vie sociopolitique des sociétés africaines depuis ce qu'on a appelé le renouveau démocratique. ${ }^{2}$ Les actrices et acteurs tant collectifs.es (partis, coalitions...) comme individuels (candidats.es, simple citoyen.e) s'appuient sur celle-ci pour défendre leurs droits et de dénoncer la violation des droits fondamentaux. Le professeur Théodor $\mathrm{Holo}^{3}$ parle d'une émergence de cette justice. Avant le changement constitutionnel de la décennie 1990 du siècle passé, dans beaucoup de pays africains la justice constitutionnelle

\footnotetext{
1 Sindjoun, Luc, Les grandes décisions de la justice constitutionnelle africaine. Droit constitutionnelle jurisprudentiel et politiques constitutionnelles au prisme des systèmes politiques africains, Bruxelles, Bruylant, 2009.

2 Adamon, Afize, Le renouveau démocratique au Bénin: la Conférence Nationale des Forces Vives et la Période de Transition, Paris, Harmattan, 1995.

3 Holo, Théodore, "Emergence de la justice constitutionnelle", Pouvoirs, Paris, n.129, 2009, p. 102.

Esta obra está bajo una Licencia Creative Commons

Atribución-NoComercial-SinDerivar 4.0 Internacional, IIJ-UNAM.
} 
était de la compétence d'une chambre des Cours suprêmes. Et dans des régimes de partis uniques, son rôle n'était pas important pour une élite, qui s'auto-légitimait et des citoyens.es, qui n'avaient pas des droits et libertés pour s'exprimer.

Depuis l'adoption des constitutions dites libérales par les pays africains au début de la décennie 1990, on observe l'importance accordée à la norme suprême de l'Etat, mais aussi le constitutionnalisme ${ }^{4}$ comme support des rapports politiques et l'idée d'un Etat de droit, qui régirait non seulement les relations entre les institutions judiciaires et politiques de l'Etat, mais aussi celles qui existent entre l'Etat et les citoyens.es.

Juristes et politologues mettent en exergue l'évolution socio-juridique et politique des sociétés africaines. La lutte politique s'est déplacée et s'appuie sur l'institution juridictionnelle, ${ }^{5}$ qui par le nouveau contexte sociopolitique, a repris des nouvelles compétences. Elle impose le respect des normes constitutionnelles et législatives aux parties dans un conflit politique et détiennent le pouvoir de proclamer le vainqueur. Mais dans les nouveaux régimes, elle recouvre un rôle qu'elle n'a pas eu jusqu'à là: la résolution des conflits politiques.

L'analyse de la justice constitutionnelle nous amène à des questions culturelles et de pratiques juridiques. Certains constitutionnalistes européens, et français en particulier, ceux qu'on a appelés "les pèlerins constitutionnels", ${ }^{6}$ ont mis l'accès sur le mimétisme. Selon eux les nouvelles constitutions seraient une copie de celles qui existent dans les vieilles dé-

4 Sur le constitutionnalisme dans les pays d'Afrique, surtout francophone, le professeur Ismaïla Madior Fall centre son analyse sur Le pouvoir exécutif des Etats d'Afrique dans Le Pouvoir exécutif dans le constitutionnalisme des Etats d'Afrique, Paris, L'Hamattan, 2008. Alors le professeur Jean-Nazaire Tama procède à une analyse historico-juridico-constitutionnelle dans L'Odysée du constitutionnalisme en Afrique, Paris, l'Harmattan, 2015. En fait les deux auteurs étudient principalement les pays d'Afrique francophone. En observant le fonctionnement des systèmes politiques d'Afrique francophone, en reprenant les termes de Tama, le constitutionnalisme se trouve se trouve à la fois dans "la voie désespoir et celle de l'espérance", pp. 20 et 21.

5 L'institution juridictionnelle n'est pas une institution nouvelle dans l'ordre institutionnel de beaucoup de pays africains. Au Bénin, sous l'ancien régime, il existait une chambre constitutionnelle au sein de la Cour Suprême. Mais dans d'autres pays, il n'y avait pas de cour, mais un conseil constitutionnel.

6 Doradeu, Renaud, "Les pèlerins constitutionnels. Éléments pour une sociologie des influences juridiques", in MENY, Yves, Les politiques du mimétisme institutionnel: la greffe et le rejet, Logiques politiques, Paris, L'Harmattan, 1993, p. 83. 
mocraties occidentales, autrement dit les constituants africains reproduiraient des institutions marquées par des cultures différentes des leurs. Or au-delà du mimétisme, ne faudrait-il pas voir un certain pragmatisme? ${ }^{7}$

Cette étude fait suite à des multiples travaux scientifiques généraux ou monographiques publiés à la fin des années 1990 jusqu'à nos jours portant sur le constitutionnalisme africain et la justice constitutionnelle. Elle n'a pas la prétention d'introduire un élément nouveau dans l'étude de la justice constitutionnelle. L'objet poursuivi est beaucoup plus modeste, il s'agit d'analyser la résolution des conflits juridiques et politiques par l'organe constitutionnel djiboutien: le conseil constitutionnel. Depuis l'adoption de la constitution libérale de 1992, l'opposition politique recourt à la justice constitutionnelle pour rechercher des solutions par la voie institutionnelle plutôt que par le recours à la violence. Un tel recours marque l'intégration des actions des acteurs sociopolitiques dans le nouveau système institutionnel.

Mais avant d'analyser le rôle de la justice constitutionnelle dans ladite résolution, il convient de répondre à certaines questions pertinentes dans une telle analyse: qu'est-ce qu'un conflit juridique et politique? Qu'estce qu'une opposition sur les résultats électoraux? Comment interpréter le refus d'une manifestation de l'opposition? Quid la modification de la constitution?

\section{NÉCESSITÉ OU MODE DE LA JURIDICTIONCONSTITUTIONNELLE}

Poser en termes de nécessité ou de mode la juridiction constitutionnelle, c'est une manière de cerner l'importance de l'institution dans un système politique de parti unique en transition vers un autre système. La nécessité

7 L'existence d'une telle institution et son fonctionnement constituent des moyens pour la garantie constitutionnelle des droits et libertés fondamentales affirmés dans les textes constitutionnels et les traités internationaux et régionaux. Elle ne serait pas effective sans une telle institution juridictionnelle, qui veille au respect des nouveaux principes constitutionnels. Mais son importance n'est pas limitée seulement à ce point; elle est garante de la suprématie de la constitution, c'est-à-dire du respect des législateurs et gouvernants dans leurs activités: adoption des lois ordinaires et des actes administratifs, et ce pour la réalisation de l'Etat de Droit; c'est-à-dire le respect du droit par les acteurs politiques et les administrations étatiques nationales et régionales. C'est donc un élément nécessaire dans un régime démocratique parce qu'elle a une légitimité essentielle dans un tel régime.

Esta obra está bajo una Licencia Creative Commons

Atribución-NoComercial-SinDerivar 4.0 Internacional, IIJ-UNAM. 
serait la mesure de son importance dans un contexte national nouveau où le pluralisme politique est un fait alors que la mode ${ }^{8}$ serait le fait d'un suivisme et donc sa relativité dans le système politique et social du pays. Dans leurs analyses, les juristes comme les politologues se basent non seulement sur des institutions et sur des actes, mais aussi sur d'autres facteurs comme l'histoire des institutions traditionnelles et modernes à la base d'un système juridique et politique, qui n'est pas immuable, et qui donne une pratique et sur la culture du peuple.

Mais se pose la question de la légitimité. Quelle est sa légitimité? Ce n'est pas l'objet de cette modeste réflexion, mais le fait d'avoir une cour constitutionnelle ou un conseil constitutionnel nous amène à ce type de questions. La recherche de la légitimité par la légalité devient théoriquement la norme dans les nouveaux systèmes politiques africains.

L'institution juridictionnelle ne s'impose pas aux constitutionnalistes et aux hommes politiques. ${ }^{9}$ Plus de trois décennies ans après les réformes constitutionnelles et politiques, l'importance et son rôle dans la vie juridique et politique d'une telle juridiction est plus que démontrer dans tous les pays. En fait, il est lié à la timide émergence d'un Etat de droit et donc au développement du droit, et en particulier le droit constitutionnel dans les Etats africains et le respect par certains Chefs d'Etat ${ }^{10}$ comme les ac-

8 Le mot mode a été utilisé par le Président Hassan Gouled Aptidon en 1991 lors de la crise sociopolitique de la République de Djibouti. En effet, pressionné par la France de suivre le changement entrepris par les autres pays africains francophones, surtout de l'Afrique de l'Ouest, après décidé de créer une commission de réforme politique, il avait que "maintenant, nous suivons la mode". Autrement, son gouvernement fera la même chose que les autres gouvernements africains: entreprendre un changement politique. Et c'est dans ce sens que j'emploie le mot "mode".

9 Favoreu, Selon Louis, "La légitimité de la justice constitutionnelle va découler en premier lieu, de l'adoption du modèle choisi au contexte institutionnel et politique". Voir l'article "La légitimité du juge constitutionnel", Revue Internationale de Droit Comparé, Paris, vol. 46, no. 2, 1994, p. 557. Comment créer un consensus des acteurs de la classe politique sur une telle institution?

10 Les trois dernières années ont été marquées par la violation des normes constitutionnelles, et surtout un point particulier, la révision constitutionnelle visant à supprimer la limitation des mandats. Dans les pays africains francophones, la procédure prévue par les constitutions n'a été respectée. Sauf au Rwanda, il n'y a pas de référendum constitutionnel. Le pouvoir exécutif, mieux la volonté du président comme au Burundi, s'est imposé pour supprimer la disposition constitutionnelle relative au nombre de mandats présidentiels d'un président en exercice. D'autres ne veulent pas respecter le calendrier électoral comme le président Kabila de la République Démocratique du Congo. 
teurs.es politiques -même s'ils ne respectent pas scrupuleusement les normes constitutionnelles - et ceux de la société civile, qui s'en remettent à l'organe constitutionnel pour résoudre leurs conflits politiques. Par ses décisions et ses interprétations quand elle est consultée, la justice constitutionnelle favorise le développement du droit constitutionnel du pays.

Comme toute autre institution politique et sociale, la justice constitutionnelle a occupé la réflexion d'abord des juristes et ensuite d'autres spécialistes en sciences humaines et sociales. Ainsi le constitutionnaliste italien Mauro Cappelletti posait la question de la nécessité pour expliquer l'importance de cette justice en affirmant que "l'existence d'une justice constitutionnelle s'impose à l'époque actuelle; elle équilibre la croissance considérable et inquiétante du législatif et exécutif; elle découle nécessairement de l'expansion des déclarations des droits". ${ }^{11}$

Pour sa part et analysant les droits constitutionnels étrangers, Stéphane Pierre-Caps affirme que "le droit constitutionnel correspond à un besoin permanent, qui est celui de l'ordre et dont le but est d'éviter l'anomie, c'est-à-dire l'absence de règle". ${ }^{12}$ La base d'un tel droit est la constitution ou la loi fondamentale que le constituant a votée, et que parfois, le peuple s'est prononcé dans un référendum.

Mais son développement ne peut avoir un impact dans les relations politiques et sociales que si les normes contenues dans la constitution sont effectives ou, selon Célestin Kentcha Tchapnga, constituent "des références majeures dans la vie politique". ${ }^{13}$ Elles sont désormais perçues comme le fondement de toute activité étatique, le gage de stabilité institutionnelle, politique et sociale. En cas de crises politiques, gouvernants et gouvernés s'y attachent très souvent en y cherchant les règles de conduite, tant sociales qu'individuelles et l'intervention du juge constitutionnel contribue à pacifier la vie politique.

Le titre de cette partie soulève des questions importantes tant juridiques que politiques. Quel est l'intérêt d'avoir une justice constitutionnelle? Un système politique pluraliste peut-il fonctionner sans une telle justice? Ces

11 Cappelletti, Mauro, "Nécessité et légitimité de la justice constitutionnelle", Revue Internationale de Droit Comparé, Paris, vol. 33, n. 2, 1981, p. 625.

12 Pierre-Caps, Stéphane, Droits constitutionnels étrangers, Paris, Presses Universitaires de France, 2010, p. 14.

13 Keutcha Tchapnga, Célestin, "Droit constitutionnel et conflits politiques dans les États francophones d'Afrique noire”, Revue Française de Droit Constitutionnel, Paris, $n^{\circ} .63,2005$, p. 451.

Esta obra está bajo una Licencia Creative Commons

Atribución-NoComercial-SinDerivar 4.0 Internacional, IIJ-UNAM. 
questions nous conduisent à l'étude d'une institution, qui est amenée à jouer, selon les juristes, un rôle fondamental dans "les nouvelles relations" entre d'une part les forces politiques et d'autre part entre celles-ci et la société civile. Ce qui soulève la nécessité de son introduction dans le système politique, mais aussi sa réception sociale, c'est-à-dire le recours des actrices/acteurs non politique de la société aux mécanismes constitutionnels.

En effet, par rapport à l'ancien contexte constitutionnel, on peut affirmer qu'il y a une nouvelle perception de la justice constitutionnelle. Par leur fonction, le juge constitutionnel ou les membres de cette juridiction fait l'objet d'une procédure de nomination particulière, suivant le modèle français et celui de certains pays d'Afrique subsaharienne d'expression francophone. Pour accomplir sa mission et ses fonctions, la constitution et la loi organique no. 4/AN/93 du 7 avril 1933 ont prévu des rôles et des attributions. ${ }^{14}$

\section{CHANGEMENT RELATIF DE SA PERCEPTION DANS LE NOUVEAU RÉGIME POLITIQUE}

La complexité du système politique et la défense des droits et libertés, et surtout le respect des principes proclamés dans la constitution conduisent aux constituants des années 1990, sans doute par l'influence extérieure, à la mise en place d'une institution dont le principal objet serait de surveiller le respect desdits droits, libertés et principes.

Aussi par l'institutionnalisation de la justice constitutionnelle, le constituant djiboutien introduit deux nouveaux contentieux dans le système juridique djiboutien: les contentieux électoral (législation électorale) et constitutionnel (respect de la suprématie de la constitution sur des normes inférieur et des droits fondamentaux par les pouvoirs publics). On assiste

14 Il nous paraît important de distinguer les attributions et les fonctions. Si dans le langage courant, il y a parfois confusion entre les deux termes, ils se manifestent différemment sur juridique et ont des conséquences différentes. Les attributions se manifestent par l'adoption des décisions juridiques, que s'imposent par exemple une décision adoptée à la suite du dépôt d'une requête sur l'organisation et/ou les résultats législatives ou présidentielles alors que les fonctions se traduisent par des avis, qui interprètent des mesures relatives à des circonstances exceptionnelles par le Chef de l'Etat veut adopter pour résoudre une situation. 
à une transposition d'une institution dans la culture juridique et politique djiboutienne. Or la transposition ne s'impose pas; elle est confrontée à de multiples facteurs culturels et sociopolitiques tant de la classe politique comme de la société. Or pour son incorporation, il faut comme l'affirme avec raison Louis Favoreu que "le contexte d'insertion s'y prête et accepte, en quelque sorte le mécanisme mis en place". ${ }^{15}$

Dans la récente histoire politique de la République de Djibouti, une telle institution n'est pas une nouveauté institutionnelle en soi. En effet l'article 3 de la loi constitutionnelle no. 2 avait expressément prévu la création d'un comité constitutionnel, composé de représentants du pouvoir exécutif, du pouvoir législatif et du pouvoir judiciaire et par de personnes désignées en raison de leur compétence. Selon Ali Mohamed Afkada, ${ }^{16}$ il est consulté pour avis sur les projets de lois organiques. La loi organique no. 1 du 10 février 1981 sur l'élection présidentielle définit ses attributions en matière électorale. La première différence qu'on peut noter entre le comité constitutionnel de 1981 et le conseil constitutionnel, prévu par la constitution du 4 septembre 1992, est l'impact d'un contexte sociopolitique international où le pouvoir politique est de plus en plus soumis à un contrôle juridictionnel. ${ }^{17}$ C'est à la suite d'une expertise extérieure que la justice constitutionnelle est devenue une institution importante dans le

15 Favoreu, Louis, "La légitimité du juge constitutionnel", Revue Internationale de Droit Comparé, Paris, $\mathrm{n}^{\circ}$. 46-2, avril-juin 1994, p. 557.

16 Afkada, Ali Mohamed, Présentation générale du Conseil Constitutionnel de la République de Djibouti, Paris, ACCPUF, Bulletin, $n^{\circ}$. 2, mai, 2001. Selon cet auteur, le conseil aurait accompli son rôle d'arbitre et de juge électoral, mais aussi d'organe consultatif pour le gouvernement. Cependant il n'évoque pas les décisions de ce comité en matière électorale et les consultations du gouvernement et se limite dire que "le Comité constitutionnel a fonctionné jusqu'en 1993».

17 Si l'institution juridictionnelle n'est donc pas un fait nouveau dans le système politique djiboutien, son rôle et son fonctionnement ne soulèvent pas moins des questions intéressantes tant au niveau politique qu'au niveau juridique pour la protection des nouveaux droits et libertés de la personne humaine. Sur le plan politique, son existence et son rôle ne sont plus à démontrer dans un régime, qui se veut démocratique. Son existence renforce la nouvelle idée qui est rentrée dans le langage politique djiboutien: 1'Etat de droit, dans le sens tout au moins théorique de la soumission au droit positif aux différents actes de l'Etat comme de ses structures. L'effectivité de l'Etat de droit se mesure au sort quotidien non seulement aux décisions d'une justice indépendante de toute pression politique et sociale, mais aussi aux décisions du juge constitutionnel.

Esta obra está bajo una Licencia Creative Commons

Atribución-NoComercial-SinDerivar 4.0 Internacional, IIJ-UNAM. 
nouveau système politique. La deuxième différence repose sur leur composition. ${ }^{18}$

Le titre de cette sous-partie nous conduit à la question suivante: qu'estce qu'institutionnaliser? Résulte-t-elle d'une évolution institutionnelle et politique?

\section{L'évolution de la justice constitutionnelle djiboutienne}

Comme les sociétés humaines, les institutions sont soumises à une certaine évolution. En fait, elles suivent le développement sociohistorique des sociétés auxquelles elles doivent produire un impact sur plusieurs plans.

Reprenant le système de la $4{ }^{\text {ème }}$ République française, les constituants djiboutiens à la fin des années 1970 ont repris l'ancien système juridictionnel français, mais en l'adaptant au contexte du nouvel Etat. Les autorités politiques ont adopté des lois dites lois constitutionnelles à partir du 27 juin 1977, date de l'indépendance, auxquelles elles ont ajouté des lois organiques. Avec ces premières lois, les autorités du nouvel Etat ont mis en place des institutions, l'une d'elles est la création d'un Comité Constitutionnel. Sa composition était plus diversifiée que l'actuel conseil constitutionnel. Aussi des réformes ont été prévues, mais elles ne se sont jamais réalisées.

En 1992 le contexte sociopolitique djiboutien est différent; le pluralisme est théoriquement instauré depuis l'adoption de la constitution du 4 septembre 1992. L'ancien comité constitutionnel est remplacé par un Conseil Constitutionnel. Il est une des nouveautés institutionnelles non seulement en matière électorale (par la régulation de la vie politique nationale), mais aussi en matière de contrôle de conformité des lois ordinaires à la constitution, autrement dit la garantie de la primauté de la constitution, et la garantie des droits constitutionnels reconnus aux personnes dans un procès (possibilité offerte à un justiciable de soulever la question de l'inconstitutionnalité d'une loi durant le procès) mais aussi la possibilité de constater l'empêchement provisoire ou définitif du président de la République. Il ne s'agit donc pas seulement d'une simple modification

18 Le Comité Constitutionnel était composé des membres nommés exclusivement par le Président de la République. Ces membres étaient désignés des différents milieux: juridique, politique, économique, religieux. Plus que des juges, ils étaient aussi des conseillers au Président de la République. 
de nom d'une institution, sinon d'une augmentation de ses compétences par rapport à la situation antérieure. Pour reprendre les propos de l'actuel président du Conseil constitutionnel, il n'est pas un organe politique mais une juridiction, qui a pour principale mission d'assurer le respect de la hiérarchie des normes et de réguler la vie politique nationale.

Entre la première et la deuxième République (après 1992), il y a eu non seulement un développement institutionnel comme d'autres pays africains, mais on a assisté à un processus d'institutionnalisation de nouveaux organes juridiques, juridictionnels, politiques et sociaux.

\section{Reflet d'une importation institutionnelle}

Pour simplifier un concept complexe on peut dire que l'institutionnalisation est le fait de donner à un rang d'institution ayant des attributions importantes et indispensables pour le fonctionnement et l'équilibre d'un système juridico-politique à un organe, qu'il soit nouveau ou ancien. Mais institutionnaliser fait penser à un autre aspect, plus important que la simple création d'une institution, il s'agit des compétences respectées par les principaux acteurs sociopolitiques. S'agissant de l'institutionnalisation de la justice constitutionnelle, il faut noter le manque de volonté des constituants postcoloniaux de certains pays africains de ne pas prendre en considération un phénomène connu dans les démocraties consolidées du Nord.

L'existence ou non d'une telle institution résulte du développement sociopolitique du pays, mais aussi de l'influence de facteurs extérieurs. Comme d'autres pays africains, l'institutionnalisation d'un parti unique en 1981 a été un grand obstacle à tout développement d'une justice constitutionnelle en République de Djibouti. L'autoritarisme et la dictature ne sont pas des régimes où la justice constitutionnelle a le droit d'être cité. L'histoire politique des anciens pays communistes de l'Europe centrale et orientale et des régimes de partis uniques du Tiers-Monde illustre cette affirmation.

\section{Ses rôles}

Avoir un rôle pour un personnage ou une institution, c'est intervenir dans des situations déterminées par le cadre juridique fondateur. Ces rôles peuvent être effectifs ou symboliques. Quant aux attributions, ce sont des 
fonctions exclusives qu'exerce une personne par son statut professionnel ou une institution. Aucune autre personne ou institution ne peut prend les actes dérivant de ces attributions.

Mais dans la pratique, on peut dire qu'il y a une certaine confusion entre attributions et rôles pour un conseil constitutionnel dont ses compétences sont bien circonscrites, qui se s'auto saisit pas à la différence d'une Cour constitutionnelle, qui a des marges de manœuvres plus larges. Le constituant de 1992 a chargé deux missions importantes au conseil constitutionnel: le contrôle et la régularité des principes constitutionnels.

Les rôles attribués par conseil constitutionnel sont prévus par un ensemble de dispositions constitutionnelles, le titre VIII de la constitution, il est composé de six articles (art.75 à 81) et la Loi Organique $\mathrm{N}^{\circ} 4 / \mathrm{AN} / 93 / 3 \mathrm{e}$ $\mathrm{L}$ du 7 avril 1993 fixant les règles d'organisation et de fonctionnement du Conseil constitutionnel.

Quels sont donc ces rôles? L'article 75 résume en 4 points ses différents rôles:

- Veiller au respect des principes constitutionnels.

- Contrôler la constitutionnalité des lois. C'est faire respecter la primauté de la constitution et le cadre constitutionnel du pays par le contrôle des lois qui lui sont soumises aux autorités politiques ou des saisines de la Cour Suprême par le contrôle d'inconstitutionnalité par exception.

- Garantir les droits fondamentaux de la personne humaine et les libertés publiques. Son existence même et son fonctionnement doivent servir une garantie pour que ces droits soient respectés et soient effectifs.

- Réguler le fonctionnement des institutions et de l'activité des pouvoirs publics.

Le contrôle et la régularité sont deux domaines importants pour une juridiction constitutionnelle. Le contrôle permet, si une loi, une mesure administrative, une norme réglementaire adoptée par une autorité compétente ou une, qui n'est pas compétente, au conseil constitutionnel sa constitutionnalité, c'est-à-dire si elle respecte la norme suprême, la constitution. Mais ce contrôle est limité et peu efficace, parce qu'il intervient à posteriori et il ne peut avoir de contrôle si le conseil n'est pas saisi par les 
autorités ou la possibilité ouverte à un/e justiciable prévues pour un tel contrôle.

Tandis qu'il y a régularité après un disfonctionnement des institutions ou encore qu'un pouvoir intervient dans le domaine exclusif d'un autre pouvoir. Le conseil constitutionnel se comporte comme le régulateur des institutions, non pas qu'il dicte les actes à prendre, mais plus chercher l'équilibre des pouvoirs pour un fonctionnement conforme à l'ordre constitutionnel. C'est une manière de rechercher une stabilité politique et sociale afin de réduire une tension politique.

Par les rôles précités, on observe une évolution de cette institution par rapport au Comité Constitutionnel de l'ancien régime politique dont le rôle était exclusivement limité aux questions électorales. Le conseil constitutionnel est à la fois un juge du contrôle constitutionnel (compétence exclusive), juge électoral (compétence partagée avec d'autres institutions) et juge du contentieux électoral (compétence exclusive) tout en étant un organe régulateur de l'activité des pouvoirs publics.

Le contexte tant national qu'international dans lequel il intervient est différent de celui de juin 1977, lequel n'a pas favorisé le développement d'une juridiction constitutionnelle. Toutefois le rôle central joué par le conseil constitutionnel, prévu par la constitution du 4 septembre 1992, ne serait effectif s'il ne s'accompagnait pas par l'exercice des attributions exclusives tant juridictionnelles que consultatives sur certaines questions institutionnelles et politiques importantes. Les rôles prévus par ladite constitution sont indicatifs. Or l'important, c'est l'exercice qui découle des rôles et des attributions.

Une de celles-ci, la plus importante, consiste à veiller la régularité de toutes les élections et les opérations de référendum. Suivant les articles 77 de la constitution et 69 de la loi organique relative aux élections du 29 octobre 1992, c'est une compétence exclusive; qui inclut aussi la proclamation des résultats électoraux, l'examen et le jugement des requêtes déposées par les partis politiques et les candidats aux élections à propos des fraudes et autres irrégularités qu'ils soulèvent. Dans un régime pluraliste le respect de la légalité pendant la compétition électorale est une obligation à laquelle tous les acteurs politiques doivent se soumettre, parce qu'elle implique le principe d'égalité, qui est un principe constitutionnel fondamental. La constitution pose un cadre général aux nouvelles institutions, les compétences de celles-ci sont souvent déterminées par une 
loi organique. Le constituant a préféré ce type de lois plutôt qu'une loi ordinaire, parce qu'il est difficile de les modifier. L'art.78 de la constitution prévoit la consultation du conseil constitutionnel par le gouvernement avant l'adoption d'une telle disposition, il y a consultation aussi en cas de modification d'une loi organique comme cela a eu lieu à propos de modifications de deux articles de la loi organique relative aux élections. En matière d'organisation des élections législatives, présidentielles et régionales et en matière de contentieux électoral, deux lois organiques posent un cadre juridique et déterminent l'institution compétente, la première est adoptée le 29 octobre 1992 (elle est relative aux élections) et la seconde le 7 avril 1993 (elle fixe les règles d'organisation et de fonctionnement du conseil constitutionnel).

\section{Ses attributions}

La définition des attributions des nouvelles institutions est précise. Le titre VIII de la constitution de 1992, composé de 8 articles (art. 75 à art. 82), est consacré au conseil constitutionnel.

La constitution du 15 septembre 1992 pose des attributions précises aux nouvelles institutions. Différentes lois organiques adoptées à partir d'octobre 1992 détaillent leurs attributions et leurs compétences. Il faut distinguer ce qu'on peut appeler les attributions actives et les attributions passives.

Par attributions actives, nous faisons référence à des interventions dont ses décisions s'imposent aux autorités. C'est le cas des décisions prises par l'institution constitutionnelle quand il est saisi par des autorités et à des moments déterminés par exemple après l'adoption d'une loi par l'Assemblée Nationale et avant sa promulgation par le Président de la République. Dans cette situation, c'est lui qui déclare si la loi est ou non conforme à la constitution. Ses décisions aux requêtes déposées par un/e candidat/e ou un parti politique après la proclamation des résultats électoraux. Ces décisions s'imposent à tous les pouvoirs de l'Etat.

Ces attributions sont renforcées par la notion de la chose jugée, c'està-dire que ses décisions ne donnent pas lieu à un appel ou recours auprès d'une autre juridiction. C'est le cas des décisions rendues suite aux requêtes déposées par les candidates/candidats et les coalitions ou partis 
politique après la proclamation des résultats électoraux. C'est qui renforce son rôle de régulatrice des institutions et des activités politiques.

Par passives, il faut entendre les avis et consultations que donne le Conseil Constitutionnel aux autorités, et particulièrement du Président de la République et du Premier ministre dans trois situations différentes.

La première attribution est relative à l'organisation d'un référendum, et en particulier des opérations du référendum selon l'art. $32 .{ }^{19}$ Cet article ne pose pas la saisine du conseil constitutionnel comme une obligation. Mais à la différence d'une élection législative ou présidentielle, un référendum est un moyen qui permet de modifier l'ordre constitutionnel. Donc comme organe régulateur, la saisine de cet organe s'impose aux pouvoirs politiques.

La deuxième porte sur l'empêchement du Président de la République ou d'un/e candidat/e lors d'une élection présidentielle, ce sont ou le Premier ministre ou le Président de l'Assemblée Nationale qui saisit le Conseil Constitutionnel selon l'art. $31^{20}$ de la constitution. Comme dans la situation antérieure, un empêchement du premier personnage de l'Etat a un impact dans la vie politique. Quant au/à la candidat/e, c'est par exemple la mort subite surtout pour les élections présidentielles.

Enfin la dernière situation, c'est la consultation pour circonstances exceptionnelles, prévue par l'art. 40 de la constitution. Seul le Président de la République peut demander au Président dudit conseil un avis dans des circonstances exceptionnelles dans le cas de l'interruption du fonctionnement des institutions, une menace de l'indépendance de la nation et de l'intégrité territoriale. Le président de la République est la seule autorité politique qui puisse agir pour rétablir la situation constitutionnelle, et pour cela il adopte des mesures. Cependant, celles-ci ne peuvent servir un moyen pour modifier "l'équilibre" institutionnel d'où l'utilité de la consultation. Son but est d'éviter un abus de pouvoir de la part de son auteur.

Depuis l'entrée en vigueur de la constitution du 15 septembre 1992, le Président de la République n'a pas saisi l'institution constitutionnelle alors

19 Article 32 Le président du Conseil constitutionnel est consulté par le président de la République sur l'organisation des opérations de référendum. Il est avisé sans délai de toute mesure prise à ce sujet.

20 Article 31 Le Conseil constitutionnel peut être saisi par le Premier ministre ou le président de l'Assemblée nationale pour constater l'empêchement du président de la République ou d'un candidat à l'élection présidentielle. Dans ce cas il statue à la majorité absolue des membres le composant dans les trois jours. 
que la situation politique était sous tension avec un blocage des institutions politiques. $^{21}$

Cependant il a une attribution, qui peut être qualifiée de sui generis, parce qu'elle peut être active, dans le sens où le processus abouti à une saisine ou non, parce qu'une autre institution interrompt la procédure. C'est le cas de l'art. 80 de la constitution du 15 septembre 1992, qui permet à une/un justiciable de soulever l'inconstitutionnalité d'une loi lors d'un procès judiciaire.

Les recours et saisines au conseil constitutionnel des autorités et formations politiques répondent à des enjeux juridiques et politiques des différents acteurs de la vie politique et sociale de la société djiboutienne.

Le développement d'un certain Etat de droit, tout au moins théorique favorise la résolution des conflits politiques, essentiellement électoraux ou liés aux élections. L'illustration de la résolution est l'adoption des décisions sur plus de 20 ans, ce qui a donné une jurisprudence du conseil constitutionnel en matière de résolution des conflits.

L'existence d'un cadre juridique et d'un Etat de droit ne suffissent pas à cerner le conflit juridique et politique, qui se pose dans les régimes libéraux. Qu'est-ce qu'un conflit juridique et politique? Une opposition sur les résultats électoraux? Le refus d'une manifestation de l'opposition? La réforme-modification de la constitution?

\section{LES ÉLÉMENTS CONSTITUTIFS D'UN CONFLIT JURIDIQUE ET POLITIQUE}

Ces questions précédemment posées ne définissent pas un conflit juridique et politique. Aucune disposition de la constitution ne fait référence explicitement à un conflit juridique ou un conflit politique. Mais on peut affirmer qu'elles manifestent ses causes ou élément d'un conflit juridico-politique.

Cependant certains juristes comme Célestin Kentcha Tchapnga propose comme définition les:

21 En 2013 après la publication des résultats des élections législatives du 22 février 2013, l'opposition avait refusé de siège à l'Assemblée Nationale. La législature a commencé à légiférer avec les représentants.es de la coalition de la majorité présidentielle. 10 sièges obtenus par la coalition de l'opposition sont vides. Aussi celle-ci a "créé" un Parlement légitime où les 10 sièges étaient réservés à la majorité présidentielle. 
...antagonismes ou les combats qui ont pour enjeu la conquête, l'exercice et le contrôle du pouvoir politique par les partis, les clans, les groupes ou les individus qui entendent imposer leur domination sur leurs adversaires. Selon lui, «une observation attentive de la scène politique africaine montre que la plupart des conflits qui s'y déroulent trouvent leur origine dans les contestations post-électorales. ${ }^{22}$

Pour sa part, Canivez Patrice, partant du sens courant, relève trois caractères:

Les conflits politiques opposent des groupes au sens le plus général du terme: classes ou couches sociales, communautés ethniques ou confessionnelles, nations et nationalités, organisations politiques (partis, États, associations d'États), etc.

Les institutions étatiques sont impliquées d'une manière ou d'une autre. L'État est partie au conflit, enjeu, arbitre ou médiateur du conflit;

Un conflit politique requiert une solution politique. Par solution politique, on entend communément une solution obtenue par la discussion par opposition à l'usage de la violence. Cette solution peut être ou non trouvée. Par conséquent, l'alternative entre violence et discussion est au cœur des conflits politiques. ${ }^{23}$

Mais les deux auteurs ne prennent en considération le fait qu'un conflit politique peut avoir pour origine une interprétation, le plus souvent subjective, d'une ou des dispositions légales. Un parti ou un candidat soulève la violation d'une norme électorale, par son non-respect, qui a favorisé son rival ou encore par le refus d'accomplir ladite norme comme cela a récemment eu lieu au Gabon après la proclamation des résultats électoraux: ${ }^{24}$ le comptage bureau de vote par bureau de vote.

Un conflit peut intervenir ou surgir à partir du moment qu'il y a une opposition entre le pouvoir et l'opposition sur le respect d'une loi, de

22 Keutcha Tchapnga, Célestin, op. cit., p. 451.

23 Canivez, Patrice, “Qu'est-ce qu'un conflit politique?", Revue de Métaphysique et de Morale, Paris, no. 58, 2008, p. 163.

24 Le cas de l'opposant, Jean Ping, qui a demandé le recomptage des votes bureau de votre par bureau de vote lors des élections présidentielles du 29 août 2016. Le refus du pouvoir de procéder au recomptage est un des facteurs, à l'origine du conflit politique au Gabon. Ce refus du recomptage, qui permet de sortir des doutes sur les résultats, n'est pas propre au régime gabonais. On en trouve aussi dans d'autres pays comme la République de Djibouti. 
la constitution, d'une loi organique dans les actes de celles et ceux qui prennent une décision. Le fait de soulever la violation d'un article de la constitution ou d'une loi, par exemple la législation électorale, constitue un conflit d'interprétation d'une norme du jeu politique. Le conflit peut naître aussi de l'opposition entre celles et ceux qui participent à la nomination des membres d'une institution, par exemple la Commission Nationale Electorale Indépendante. Un tel conflit peut paralyser le fonctionnement de cette institution et avoir un impact sur les échéances électorales.

\section{LES MECANISMES DE LA RÉSOLUTIONDES CONFLITS JURIDIQUES ET POLITIQUES}

Sans mythifier l'Etat de droit, son existence et le fonctionnement régulier des mécanismes et des institutions prévus par le cadre constitutionnel ne constituent pas des éléments de garantie contre l'éclatement de toute crise institutionnelle ou d'un conflit politique. Les conflits naissent ou surgissent des disputes entre les membres de l'élite dirigeante, mais aussi dans les relations entre ceux-ci et les citoyens.es.

A la différence d'un régime autoritaire, le conflit juridique et/ou politique n'est pas nié. Le contexte constitutionnel et institutionnel prévoit des mécanismes pacifiques de résolution pour éviter l'instabilité institutionnelle. Les parties au conflit recourent à la voie juridictionnelle. ${ }^{25}$ Sa résolution suit des procédures bien déterminées. Selon le cadre constitutionnel, c'est une institution qui a des attributions et des compétences exclusives d'abord en termes de prévention par le contrôle des attributions des pouvoirs institutionnels ou les actes des acteurs.es politiques.

\section{Le contrôle des attributions et compétences entre le pouvoir législatif et le pouvoir exécutif}

Les conflits politiques sont liés à l'exercice des compétences des autorités relevant d'un pouvoir. Dans le régime de parti unique, il n'y avait aucun

\footnotetext{
25 Théoriquement, les acteurs.es politiques doivent suivre ces procédures. Mais dans la pratique, dans certains pays d'Afrique subsaharienne, on observe encore dès nos jours la réminiscence des pratiques autoritaires de l'ancien régime. Les arrestations arbitraires des opposants continuent, la justice constitutionnelle et la justice ordinaire sont confinées par le pouvoir exécutif.
} 
contrôle de compétence entre les deux principaux pouvoirs, l'exécutif et le législatif, parce qu'ils ont confondus et l'assemblée parlementaire n'est qu'une chambre d'enregistrement des desideratas du pouvoir exécutif. ${ }^{26}$ Quelques mois après la proclamation de l'indépendance, on assiste à un pouvoir personnel fort, celui du Président de la République, qui se voulait l'incarnation de la construction de l'unité nationale et évitant ainsi tout débat entre les différentes forces politiques de la coalition gouvernementale. Aucune des deux lois constitutionnelles, adoptées le jour même de l'indépendance le 27 juin 1977, ne fait référence à un contrôle de compétence entre le législatif et l'exécutif.

La constitution du 15 septembre 1992 a introduit quelques changements dans les rapports entre le pouvoir législatif et le pouvoir exécutif, qui reste prépondérant. Le Titre VI est consacré aux rapports entre le pouvoir législatif et le pouvoir exécutif. ${ }^{27}$ Et le déséquilibre du bicéphalisme du pouvoir exécutif continue. Le conseil constitutionnel et le Président de la République interviennent concurremment dans la régulation du fonctionnement des institutions. Il a un rôle de régulation fonctionnement des institutions.

Comme on a pu voir antérieurement, réguler le fonctionnement des institutions est une fonction importante du conseil constitutionnel. Mais que signifie réguler le fonctionnement des institutions anciennes et nouvelles?

26 On trouve aussi cette situation dans un régime pluraliste, qui se veut démocratique, mais dont la vie parlementaire est monopolisée par une majorité écrasante du parti présidentiel ou d'une majorité présidentielle, qui soutient le gouvernement. C'est un cas de figure, qui reproduit, un caractère du régime autoritaire parce qu'il n'y a plus de débat entre une majorité et une opposition. La ou les chambres parlementaires deviennent une simple institution d'enregistrement de la volonté du gouvernement. Depuis 1992, c'est le cas de la République de Djibouti.

27 Ces sont des rapports déséquilibrés, rationnalisant et réduisant le champ du pouvoir législatif par la détermination de ses domaines d'intervention et d'initiative prévus par 1'art. 57. Le nouveau régime, qui est en fait dans la continuité du précédent régime, est fortement présidentialiste. Il y a une concentration du pouvoir exécutif entre les mains du Chef de l'Etat. Le Premier ministre reste plus que jamais "le premier des ministres" dans l'ordre protocolaire, il n'a aucun pouvoir constitutionnel et politique. Voir Laudouze, André, Djibouti, nation carrefour, Paris, Karthala, 1982. Au début de l'ancien régime, le locataire de la Primature avait une compétence ministérielle-durant plusieurs années le troisième Premier ministre de l'ex président Hassan Gouled Aptidon avait les compétences du ministère du Port. La réforme constitutionnelle du 21 avril 2010 n'a renforcé que le pouvoir présidentiel en supprimant la limite à deux mandats présidentiels successifs. 


\section{La régulation problématique des institutions par la juridiction constitutionnelle}

En prenant la définition du Larousse, l'adjectif régulateur nous conduit aux verbes régler et régulariser. Les deux verbes font référence à une situation de conflit, qu'il faut trouver une solution. Mais en retenant le verbe régulariser, on arrive à l'action de "rendre conforme aux lois, aux règlements, mettre en règle" une situation donnée. Donc faire respecter aux autorités élues ou non élues les lois (fonctionnaires et agents des administrations publiques) et les règlements qui prévoient et posent en même temps des limites à leur autorité. Il arrive que par l'action de ces fonctionnaires et agents ayant de l'autorité publique ces règles, règlements et procédures sont violés. Et par la même occasion, cette action entraîne une vulnération des droits fondamentaux, prévus par la constitution et les traités internationaux, signés et ratifiés par l'Etat djiboutien.

Quant au mot régulation, on retiendra le deuxième point "le fait de maintenir en équilibre, d'assurer le fonctionnement correct" d'un système complexe d'interactions. Comme institution, supposée s'appuyer sur le droit constitutionnel et être neutre, le conseil constitutionnel par ses décisions régule théoriquement le fonctionnement des institutions. Même si la nature du régime politique n'a pas changé beaucoup par rapport au régime de parti unique, le législateur djiboutien confère un nouveau rôle à l'organe constitutionnel. Selon l'al. 2 de l'article 75 de la constitution, le conseil constitutionnel est un organe régulateur du fonctionnement des institutions et de l'activité des pouvoirs publics. En fait c'est une fonction très symbolique. Dans le titre Titre IV de la constitution du 15 septembre 1992 "Des rapports entre le pouvoir législatif et le pouvoir exécutif", on trouve des références dudit conseil aux articles $58,{ }^{28} 60^{29}$ et

28 Article 58 Les matières autres que celles qui sont du domaine de la loi en vertu de ladite constitution ressortissent au pouvoir réglementaire. Les textes de forme législative intervenus en ces matières peuvent être modifiés par décret si le Conseil constitutionnel à la demande du Président de la République déclare qu'ils ont un caractère réglementaire en vertu de l'alinéa précèdent. Cette article limite clairement le pouvoir législateur, et présente comme un rempart contre la violation de la compétence du pouvoir réglementaire, donc du pouvoir exécutif.

29 Article 60 Les propositions projets et amendements qui ne sont pas du domaine de la loi sont irrecevables. L'irrecevabilité est prononcée par le président de l'Assemblée nationale après délibération du bureau. En cas de contestation le Conseil constitutionnel saisi par le président de l'Assemblée nationale ou le Président de la République statue dans un délai de vingt jours. 
67. ${ }^{30}$ Ces dispositions constitutionnelles concernent les champs législatif (députés) et réglementaire (gouvernement), qui, amènent le pouvoir législatif à interférer les compétences du pouvoir exécutif. Dans le nouveau système politique djiboutien, il y a une rationalisation pour ne pas parler de minorisation du pouvoir législatif par rapport au pouvoir exécutif. En fait à la lecture des articles précités, il n'y a pas de régulation, il s'agit bien de la protection du large champ d'intervention du pouvoir exécutif. Le détenteur de ce dernier partage avec les députés, selon l'art. 59 de la constitution du 15 septembre 1992, l'initiative des lois et le droit d'amendement, ce qui a traduit la chambre parlementaire en une chambre d'enregistrement des projets adoptés par le gouvernement en conseil des ministres. Dans un tel contexte, quel type de régulateur peut-être le conseil constitutionnel?

Le rôle de régulateur de la juridiction constitutionnelle ne peut être effectif qu'à la condition que ses décisions soient respectées par les deux pouvoirs politiques: le législatif et l'exécutif. Or dans une demande du parquet en 1996 relative à la levée de l'immunité parlementaire de trois parlementaires poursuivis pour être jugés, jouant son rôle de régulateur le conseil constitutionnel constate un dysfonctionnement, qui résulte d'un non-respect de la procédure prévue par le Règlement Intérieur par l'Assemblée nationale par son principal représentant le Président de l'institution parlementaire. En effet au lieu de se conformer au dudit règlement, le président adresse une simple lettre donnant lieu à la levée de l'immunité parlementaire. Les députés ont été poursuivis illégalement et condamnés à des peines de prison et une interdiction de participation à toute activité politique durant 5 ans.

La meilleure régulation n'est-elle pas une auto-régulation des institutions par le respect de leurs compétences et celles de chacune d'elle. Mais aussi paradoxale qu'elle soit, une capacité de régulation est accordée au premier personnage de l'Etat par la constitution. Dans le nouveau constitutionnalisme après 1992, le rôle prépondérant du Chef de l'Etat est maintenu.

Mais en fait son principal rôle en matière institutionnelle est d' "assurer la continuité du fonctionnement des institutions de la République". Selon

30 Article 67 Les lois auxquelles la constitution confère le caractère de lois organiques ne peuvent être adoptées qu'à la majorité absolue des membres de l'Assemblée nationale et ne peuvent être promulguées qu'après déclaration par le Conseil constitutionnel de leur conformité avec la constitution. 
les termes de la deuxième phrase du $2^{\text {ème }} 1$ 'alinéa de l'art. 4. C'est une formula laconique. Mais la mauvaise formulation de la phrase laisse penser que le fonctionnement des institutions du pays dépend de ce personnage. Le législateur de 1977 n'a posé aucune précision, ni aucune limite aux actions qu'il peut entreprendre pour assurer le fonctionnement des institutions. Par ailleurs, cette action n'est liée à aucune circonstance. C'est un champ libre laissé à sa volonté. Finalement il reste le maître qui décider les actes et les mesures à prendre pour la continuité d'une institution, qui ne fonctionne pas.

Lors du changement politique de 1992, le constituant a repris la même posture qu'en 1977. Le nouveau régime place le chef de l'Etat au centre de la vie institutionnelle et politique du pays. Ainsi selon l'article $22 \mathrm{du}$ Titre III du Président de la République de la constitution du 15 septembre 1992, "il incarne l'unité nationale et assure la continuité de l'Etat". Cependant l'art. 40 de ladite constitution, le législateur a introduit un changement. Il n'est plus question d'assurer la continuité d'une institution, mais de l'Etat (art. 20) et du "fonctionnement régulier des pouvoirs publics est interrompu" (art. 40). Même s'il reste le maître à décider les mesures à prendre, cependant il peut s'appuyer sur l'avis du président de l'Assemblée nationale et du président du Conseil constitutionnel et après en avoir informé la nation par un message (art. 40) "prendre toute mesure tendant à rétablir le fonctionnement régulier des pouvoirs publics et assurer la sauvegarde de la nation, à l'exclusion d'une révision constitutionnelle". Ce n'est pas une obligation constitutionnelle l'avis des présidents de l'Assemblée Nationale et du Conseil Constitutionnel. En fait le verbe pouvoir n'implique pas une obligation constitutionnelle, mais peut-être politique au Président de la République. La mesure adoptée est de "nature législative" et donc l'Assemblée nationale doit être saisie pour la ratifier ou la rejeter, puisqu'elle est devient un projet de loi.

Plus qu'une simple régulation du fonctionnement des institutions, le rôle prépondérant du Chef de l'exécutif peut constituer un moyen de contrôle de leur fonctionnement, ce qui renforce sa domination politique sur les autorités politiques.

Donc si le conseil constitutionnel partage avec le chef du pouvoir exécutif, la régulation des institutions, le constituant de 1992 a reconnu une compétence exclusive à l'organe constitutionnel pour régler tout conflit juridique par plusieurs voies. Un conflit peut avoir à la fois un aspect ju- 
ridique, par exemple par la violation d'une loi, et se transformer en un conflit sur les élections, et donc c'est la voie du contentieux électoral que les acteurs doivent chercher la solution.

\section{LA COMPÉTENCE EXCLUSIVE EN MATIÈRE DE CONTENTIEUX ÉLECTORAL}

Dans l'ancien régime politique, ${ }^{31}$ il y avait bien une référence au contentieux électoral. Mais il n'y a pas eu une contestation électorale par la nature même dudit régime: la monopolisation de la vie politique par un parti unique ne favorise pas le développement d'un contentieux électoral. Car le parti gouvernemental présentait un candidat unique aux élections présidentielles et une liste des candidats de la formation politique unique.

Dans le régime politique de 1992, cette institution est sortie de son isolement. En effet comme on a pu voir dans les sections précédentes, elle a eu des compétences exclusives parmi lesquelles celle de juger tout contentieux relatif à l'organisation des élections (nationales, régionales et locales) et les contestations quant à leurs résultats. Selon l'article 69 de la loi organique $n^{\circ} 1 / \mathrm{An} / 92$ relative aux élections: Le contentieux de toutes les élections relève de la compétence du conseil constitutionnel. Cette compétence est reconnue aussi par la loi organique $\mathrm{n}^{\circ} 4 / \mathrm{AN} / 93 \mathrm{du} 7$ avril 1993. ${ }^{32}$

Il convient d'abord de cerner le contentieux électoral dans le nouveau jeu politique (1). Comme tout contentieux, le contentieux électoral est soumis à un formalisme et à des procédures particulières (2) que les parties doivent suivre et respecter (3).

\section{Le contentieux électoral dans le nouveau jeu politique}

Il est tautologique d'affirmer que l'organisation des élections et la proclamation de leurs résultats dans un régime où les élections plura-

31 Par ancien régime, nous faisons référence au régime mis en place le jour même de l'indépendance, le 27 juin 1977, par les lois constitutionnelles $n^{\circ} 1$ et $n^{\circ} 2$. C'est un régime fortement présidentialiste, qui adopte l'exclusion de toute opposition politique par l'institutionnalisation du parti unique en 1981 après la création d'un nouveau parti politique par le Premier ministre démissionnaire.

32 Le chapitre $\mathrm{V}$ est dédié au contentieux électoral de l'art. 38 à l'art. 48.

Esta obra está bajo una Licencia Creative Commons

Atribución-NoComercial-SinDerivar 4.0 Internacional, IIJ-UNAM. 
listes, ${ }^{33}$ constituent théoriquement un moyen d'acquisition du pouvoir, conduisent souvent à diverses contestations. Celles-ci résultent de l'absence d'une consolidation des mécanismes institutionnels, le respect des normes juridiques en vigueur par une partie. Aussi l'instauration de la démocratie électorale n'est pas la suppression de tout conflit entre les forces politiques dans la société djiboutienne. Cependant à la différence d'un régime autoritaire, le règlement des conflits juridiques et politiques relèvent de la compétence des institutions juridiques et juridictionnelles, lesquelles reposent théoriquement sur un consensus des différents acteurs, en recourant à celles-ci et en acceptant leurs décisions. Le but de celles-ci est de gérer de manière pacifique, donc par la voie juridique et politique, et ce pour préserver la stabilité apparente du nouveau système politique pluraliste en exerçant leurs attributions.

En République de Djibouti, depuis 1992, la proclamation des résultats des élections législatives et présidentielles conduit souvent à un contentieux, ${ }^{34}$ déposé au conseil constitutionnel. Dans le langage courant, on a une conception réduite de ce contentieux. Pour la majorité des politiques comme les citoyens.es, il est lié à la proclamation des résultats électoraux et la contestation qui en résulte. Mais il n'est pas limité à un aspect du processus électoral. En effet, selon le professeur Maurice Kamto, il "s'entend à la fois de l'ensemble des contestations ou de litiges liés à l'organisation, au déroulement et aux résultats des élections, et de l'ensemble des règles régissant la solution de ces litiges par voie juridictionnelle". ${ }^{35}$ Selon Francis Delprée, ${ }^{36}$ il faut distinguer deux types de contentieux, qui interviennent à des moments différents: le contentieux pré-électoral, avant le jour des élections, ${ }^{37}$ et le contentieux post-électoral, qui a lieu après la proclamation des résultats électoraux. Leurs objets sont différents.

33 L'adoption du cadre juridique électoral, le contrôle de l'administration et de l'organisme, qui supervise le processus électoral est sous le contrôle d'un parti ou d'une coalition. Les opposants des pays africains soulèvent la question de la manipulation de ce cadre par le pouvoir. Dans des régimes où le pluralisme électoral est récent dans la vie politique, le contrôle dudit processus est déterminant pour la victoire électorale.

34 Abdillahi Bahdon, Mohamed, "Note de Mohamed Abdillahi Bahdon", Revue de Droit Africain. Doctrine et jurisprudence, Bruxelles-Kinshasa, no. 31, juillet 2004, p. 417.

35 Kamto, Maurice, "Le contentieux électoral au Cameroun", Latex Lata, Yaoundé, no. 20, novembre 1995, p. 3.

36 Delprée, Francis, Le contentieux électoral, Presses Universitaires de France, Paris, 1998.

37 Le jour de l'élection culmine tout un travail législatif et administratif. L'élection 
Pour sa part, Djedjro Francisco (2009) met l'accent sur sa gestion en affirmant que "le contentieux électoral passe par une gestion difficile et son implantation est variable selon les pays". ${ }^{38}$ Selon plusieurs facteurs tant juridiques que psychologiques, les acteurs.es politiques peuvent donner moins d'importance à tel contentieux. C'est surtout le cas des pays où la transition vers le régime pluraliste s'est mal déroulée ou a été déterminée.

Le contentieux électoral ne peut être réduit seulement aux contestations soulevées par les candidats.es et les formations politiques après la proclamation des résultats électoraux. Dans la loi djiboutienne, aucun article des différentes lois, ni leurs modifications ultérieures ne fait référence à un contentieux avant le jour J. des élections. Cependant, on a noté des décisions du Conseil constitutionnel dont l'objet n'était pas lié à des contestations sur la régularité ou des résultats des élections, sinon des actes administratifs relatifs à d'autres aspects comme la composition de la liste ou le changement de position des candidats.es comme les décisions $n^{\circ} 01 / 2013 /$, $n^{0} 2 / 2013$ et $n^{0} 3 / 2013^{39}$ du Conseil Constitutionnel ${ }^{40}$ du 4 février alors que l'élection avait lieu le 22 février. En fait ces requêtes dans un processus, qui impliquent des opérations préparatoires, qui interviennent avant le jour de l'élection comme la composition des listes des partes. Le gouvernement et les institutions chargés de l'organisation des élections adoptent des

relève d'un processus qui commence des mois avant dont différentes parties, institutions administratives et formations politiques prennent des décisions. Le constitutionnaliste belge, Francis Delprée, affirme avec raison que "ces différents ne naissent pas un jourcelui du scrutin. Ils s'inscrivent dans un processus, c'est-á-dire un ensemble d'opérations — qui s'enchaînent les unes aux autres — et qui concourent à la sélection des élus", Delprée, Francis, op. cit., p. 3

38 Meledje, Djedjro Francisco, "Le contentieux électoral en Afrique", Pouvoirs, Paris, no. 129, 2009, p. 129.

39 Le conseil constitutionnel a rendu 3 décisions, déposées à des différentes dates, le même jour. Les décisions $n^{\circ} 01 / 2013$ /, déposée le 4 février 2013, ${ }^{\circ} 2 / 2013$, déposée le 30 janvier 2013et n ${ }^{\circ} 3 / 2013$, déposée le 4 février 2013. Il prend ces décisions le 4 février alors que l'élection avait lieu le 22 février. Elles relèvent d'un contentieux pré-électoral.

40 Avant 1993, c'est la Cour Suprême qui avait compétence pour décide sur le recours introduit par une/un candidate/candidat, un parti politique et/ou une coalition de partis. En effet la décision du premier recours contre les résultats des premières élections législatives du pays organisées le 18 décembre 1992 est rendue par la Cour Suprême. L'auteur dudit recours est le seul parti de l'opposition, qui avait présenté des candidats aux premières élections législatives pluralistes et libres. 
normes, qui peuvent faire l'objet de recours par les candidates/candidats, les coalitions et les partis politiques.

Cependant le contentieux électoral soulève un conflit à la fois juridique et politique, qu'il faut résoudre au plus vite pour éviter une instabilité, qui pourrait se manifester en une guerre civile comme au Kenya lors des élections présidentielles en 2008 et en Côte d'Ivoire lors des élections présidentielles de 2010. L'élection étant avant tout un acte politique puisqu'il s'agit d'un choix politique des électrices/électeurs, mais il est circonscrit dans un cadre juridique.

\section{Formalisme et procédures}

L'intérêt d'une procédure est d'informer et d'orienter celles et ceux qui recourent au droit et aux instruments juridiques prévus par le cadre législatif pour défendre leurs droits, mais aussi respecter la législation électorale quand ils sont violés par des institutions, des personnes morales et des particuliers, et ce au lieu de recourir à la violence. Sur certains aspects, la procédure peut favoriser certaines parties par rapport à d'autres par exemple les délais.

Le formalisme se manifeste d'abord par la forme du recours et de la requête. C'est par un écrit que la partie demandeuse dépose un recours. C'est une exigence légale. L'art. 38 de la Loi Organique $n^{\circ} 4 /$ $\mathrm{AN} / 93 / 3$ eme L fixant les règles d'organisation et de fonctionnement du Conseil Constitutionnel stipule que "le Conseil Constitutionnel ne peut être saisi que par une requête écrite adressée à son secrétariat". L'article 71 de la loi Organique $n^{\circ}$ 1/AN/92 du 29 octobre 1992 renforce la condition posée par l'article précédemment cité et ajoute d'autres éléments: "la requête doit être écrite. Elle est adressée au secrétariat du conseil constitutionnel ou au ministre de l'intérieur qui en assure la transmission immédiate au conseil constitutionnel. S'il s'agit d'une élection législative, le bureau de l'Assemblée Nationale est avisé du dépôt du recours".

Par ailleurs, et suivant l'art. 39, les requêtes doivent porter le nom, le prénom et qualité du requérant, les moyens d'annulation invoqués. Le requérant peut annexer à la requête les pièces produites au soutien de ses moyens. 


\section{Les parties dans le contentieux électoral}

Tout contentieux oppose au moins deux parties, qui s'opposent à une décision administrative ou judiciaire et/ou un résultat d'une élection. Mais quelle qualité doivent-elles avoir?

Le droit constitutionnel djiboutien pose des conditions pour être partie dans un contentieux électoral. La principale condition est d'être: un/e candidat/e, un parti politique et/ou coalition de partis politiques. Ce qui exclut l'action d'un/e citoyen/e contre des fraudes électorales, qui ont lieu dans un bureau de vote. Les lois organiques adoptées en $1992\left(\mathrm{n}^{\circ}\right.$ 1/AN/92 du 29 octobre 1992$)^{41}$ et 1993 ( $n^{\circ}$ 4/AN/93/3e L du 7 avril 1993) limitent les parties dans un tel procès aux candidats.es et aux formations politiques, qui ont pris part aux élections.

Mais il ne suffit d'être un/e, candidat/e, un parti politique ou coalition de partis politiques, il faut avoir un intérêt à agir. C'est qu'a soulevé le juge constitutionnel dans la décision $n^{\circ}$ 002/98/CC du 18 février 1998. Mais il ne donne pas une définition de "l'intérêt à agir". Dans cette décision, le requérant se présente comme le principal dirigeant d'un parti politique. Or son parti s'est présenté aux élections, mais avec un autre président.

Suite à une crise de son parti à la mort de son fondateur, deux dirigeants se proclament président. Suivant le cadre légal, manipulé par le pouvoir, c'est le ministre de l'Intérieur, qui accorde un certificat de reconnaissance au Président élu lors d'un congrès. Or dans le cas en question, il y a une forte opposition entre deux personnalités pour occuper la présidence du parti. Le ministre de l'Intérieur accorde le certificat de reconnaissance au concurrent requérant. Ce dernier conteste la décision du ministre.

Les candidats.es ne soulèvent pas un délit contre une personne physique, mais plutôt des infractions relatives à la législation électorale par exemple la violation des normes sur l'organisation des élections, l'empêchement de la présence des délégués des partis dans les bureaux de vote, lors du dépouillement des bulletins de vote. Celle-ci constitue une des bases de la justice constitutionnelle, la constitution étant aussi une référence pour les grands principes comme l'égalité. En fait, en soulevant les infractions, la/ le requérante/requérant met en doute le résultat des élections, et donc la

\footnotetext{
41 L'article 70 de cette loi organique stipule que "tout candidat peut intenter un recours sur la régularité des opérations électorales dans les dix jours qui suivent la proclamation des résultats du scrutin". 
victoire de ses concurrents. Elle/il soulève un conflit lié à la compétition électorale pour non-respect des principes constitutionnels comme le principe d'égalité, pour usage abusif des ressources publiques, pour abus de pouvoir (le cas du parti au gouvernement et des candidats aux législatives et du candidat aux présidentielles de ce parti).

Dans la jurisprudence du conseil constitutionnel, à part une requête, la question de l'intérêt d'agir n'est pas une question soulevée dans tous les recours. Il est reconnu dans la majorité des cas, après étude des arguments de la requête ou de la saisine, une décision est rendue.

\section{LES SAISINES DU CONSEIL CONSTITUTIONNEL COMME MOYEN} DE RESOLUTION DES CONFLITS JURIDIQUES

Les saisines constituent un moyen pour les parties de trouver une solution à leur conflit juridique, et en particulier en cas violation des droits, libertés et principes fondamentaux comme l'égalité des candidats.es et formations politiques en période électorale.

Le conseil constitutionnel est une institution juridictionnelle, qui interprète la constitution, la loi suprême du pays, face à des normes inférieures (loi, décrets) adoptés par le Parlement, l'administration centrale et les administrations (régionales et locales).

Théoriquement il intervient d'un point de vue juridique, parce qu'il doit être le garant de la stabilité des institutions du pays. Mais une tension politique peut avoir comme objet l'interprétation d'une disposition juridique, quel que soit son niveau dans l'ordonnance juridique du pays. Il s'agit de réguler la vie politique durant la période de transition. Comme affirment Luc Sundjoun et Mathias Eric Owona Nguini dans leur analyse:

...la régulation juridique de la vie politique doit se faire au regard des exigences d'Etat de droit et de démocratie. Il ne s'agit pas seulement de normaliser la politique mais aussi de transformer les manières de faire de la politique, de tracer les contours d'un nouvel ordre politique. Quant à la politisation du droit, elle renvoie aux enjeux qui déterminent le champ juridique, aux luttes entre acteurs socio-politiques en vue du monopole de la formulation des règles du jeu. ${ }^{42}$

42 Sindjoun, Luc et Nguini Owona, Mathias, "Politisation du droit et juridicisation de la politique: 1'esprit sociopolitique du droit de la transition démocratique au Cameroun", 
A la différence de la Cour Constitutionnelle du Bénin, le conseil constitutionnel djiboutien n'est pas un organe juridictionnel, qui peut position en attirant l'attention des dirigeants.es quand il observe des signes ou menaces, qui peuvent avoir un impact sur l'équilibre institutionnel ou créer une tension juridico-politique dans le pays durant une compagne électorale.

Selon la loi organique $n^{\circ} 4$ du 7 avril 1993, c'est par la saisine de dirigeants (Président de la République, le Premier ministre, 10 députés, des candidats.es, coalitions et partis politiques) et d'institutions judiciaires (Cour Suprême pour le cas exclusif d'une inconstitutionnalité soulevée par un/e justiciable lors d'un procès), qui sont habilités à le saisir. Sa principale activité est liée à donner une décision sur les requêtes déposées par ces autorités. En dehors de ces cas, il est consulté par le Présidente de la République pour les mesures à adopter dans un contexte exceptionnel, prévu la constitution du 15 septembre 1992.

\section{La saisine des députés contre une décision du bureau de l'Assemblée Nationale}

Selon le contexte constitutionnel djiboutien, la saisine des députés est limitée à la contestation d'une loi votée définitivement par le bureau de l'Assemblée Nationale; laquelle doit intervenir dans un délai de huit jours après son adoption définitive (al. 2 de l'art. 79 de la constitution du 15 septembre 1992). Il s'agit d'une décision collective. Ni la constitution, ni la loi Organique $\mathrm{n}^{\circ}$ 4/AN/93/3ème L du 07 avril 1993 fixant les règles d'organisation et de fonctionnement du Conseil Constitutionnel ne prévoient une autre possibilité pour les députés.es de saisir sur le conseil constitutionnel. Le non-respect du cadre constitutionnel entraîne un conflit politique, qui aura des conséquences sur le fonctionnement des institutions.

Or l'évolution du contexte politique peut conduire un changement sur ce point. En effet, un conflit entre personnalités politiques peut déboucher sur une saisine au conseil constitutionnel. C'est ce qui est arrivé vers la fin de l'année $1995 .{ }^{43}$ Critiqué pour sa gestion de main de fer des af-

en Du Bois de Gaudusson, Jean et Darbon, D., La création du droit en Afrique, Paris, Karthala, 1997, pp. 216-245.

43 Le public djiboutien assiste à la première crise de la direction de l'ex parti unique.

Esta obra está bajo una Licencia Creative Commons

Atribución-NoComercial-SinDerivar 4.0 Internacional, IIJ-UNAM. 
faires de l'Etat par le ministre de la Justice et Secrétaire Général du parti unique et d'autres ministres et députés de son parti, le Président de la République dépose une plainte pour diffamation au Tribunal de Grande Instance contre les auteurs. Sauf le Directeur du cabinet présidentiel, les autres ont une immunité parlementaire, et pour autant il y a une procédure à suivre tant par l'institution à laquelle ils appartiennent comme la pouvoir judiciaire. Sans respecter le règlement intérieur de l'Assemblée Nationale, le Président de l'Assemblée Nationale adresse une lettre au ministre de la Justice affirmant la perte de l'immunité parlementaire de trois députés. Lors de la procédure de vote de la levée de leur immunité parlementaire, leurs droits ont été déniés. Conformément aux stipulations de l'al. 5 de l'art. 10 de la constitution du 15 septembre 1992, leur droit à la défense n'a pas été respecté, ni celui de se faire assister par l'avocat de son choix, qui sont théoriquement garantis à tous les stades de la procédure.

Par ailleurs, et en renforçant ces droits, l'article 64.5 du règlement intérieur de l'Assemblée nationale stipule que "La commission saisie d'une demande de levée de l'immunité parlementaire doit entendre le député intéressé, lequel peut se faire représenter par un de ses collègues".

Sur le plan formel, il y a eu une violation du règlement intérieur de l'Assemblée Nationale. En effet au lieu d'une résolution du bureau de cette institution, c'est une lettre de son Président qui notifie au Procureur de la République la levée de l'immunité parlementaire desdits députés.

Face à cette décision, qui a violé la procédure à suivre, prévue par le Règlement Intérieur de l'institution à laquelle ils appartiennent, dix députés saisissent le Conseil Constitutionnel. La question de la levée de l'immunité des parlementaires n'entre pas dans ses compétences. En acceptant d'étudier le recours déposé par ce groupe d'élus nationaux, le conseil constitutionnel étend ses compétences. En fait, cela rentre dans la régulation des institutions. Il y a un conflit juridique (non-respect du cadre constitutionnel et légal) et politique (conflit entre hommes politiques) qui a un impact théoriquement sur le fonctionnement des institutions poli-

Cette crise politique n'est que l'aboutissement des tensions entre membres du parti sur la succession du patriarche de la politique djiboutienne, Hassan Gouled Aptidon. Pris d'un malaise alors qu'il participait à un sommet des Chefs d'Etat de la Francophonie à Cotonou, il est hospitalisé dans un hôpital parisien. Au lieu de respecter le cadre constitutionnel, le Chef de l'Etat repartit en suivant sa stratégie politique ses compétences entre le directeur et le chef du cabinet présidentiel. Or ce sont deux personnages, qui étaient sur la liste occulte de la succession du premier personnage de l'Etat. 
tiques (présidence et assemblée parlementaire). C'était la première fois que cet organe était saisi pour un tel conflit.

Mais aussi surprenant que cela puisse paraître, le conseil constitutionnel a accepté la saisine d'un citoyen contre une décision administrative.

\section{La saisine d'un.e citoyen.e}

Comme on a pu voir dans la deuxième parte de cette réflexion, la saisine directe d'un.e citoyen.e n'est pas prévue par la constitution du 15 septembre de 1992, ni par loi Organique n 4/AN/93/3ème L du 07 avril 1993. Cependant, et exceptionnellement, un citoyen a saisi le conseil constitutionnel le 30 septembre 1997 pour demander la levée totale ou partielle de l'immunité parlementaire d'un député, pour sa fonction de ministre de l'Intérieur et de la Décentralisation. Mais après une plainte déposée auprès Procureur Général Près la Cour d'Appel de Djibouti pour "abus de pouvoir et destruction des biens appartenant à autrui", ${ }^{44}$ lequel a adressée à l'Assemblée Nationale une demande de la mainlevée de l'immunité parlementaire dudit député, ex ministre de l'Intérieur et de la Décentralisation en date du 07 janvier 1996. L'objet de cette saisine, que le conseil constitutionnel a accepté, était de poursuivre un ex membre du gouvernement et au moment de la saisine député devant les tribunaux de droit commun pour les dommages causés à ce citoyen par un acte administratif adopté le cadre de ses compétences ministérielles.

Ce citoyen se sent lésé par l'attribution d'un terrain dont il est le propriétaire à une autre personne. Le ministre ordonne la destruction de la construction entreprise par le requérant. C'est un conflit lié au droit de propriété, un droit fondamental. Dans sa décision n $98-001 / \mathrm{CC}$ du XX, le conseil constitutionnel considère "l'excès de pouvoir et la destruction des biens appartenant à autrui dont a été victime, le requérant Monsieur Hassan Omar Farah". ${ }^{45}$ L'institution juridictionnelle ne se limite à une simple constatation, mais déclare que ces faits:

44 Considérant 9 de la Décision n 98-001/CC que "Monsieur Hassan Omar Farah déposait plainte auprès du Procureur Général Près la Cour d'Appel de Djibouti pour abus de pouvoir et destruction des biens appartenant à autrui contre Monsieur Idriss Harbi Farah”.

45 Considérant 12 de la Décision $n^{\circ}$ 98-001/CC. 
...sont imputables aux abus commis par Monsieur Idriss Harbi Farah, ex-Ministre de l'Intérieur et de la Décentralisation de l'État de la République de Djibouti, qui a méconnu le droit de propriété ainsi que les constructions de Monsieur Hassan Omar Farah qui avait été autorisées à effectuer son terrain, que cette faute est de nature à engager sa propre responsabilité et celle de l'État de Djibouti envers Monsieur Hassan Omar Farah, qu'il sera fait une exacte appréciation des faits de la cause et du montant de la réparation qui lui est dû par les Tribunaux, notamment le Conseil du contentieux administratif de Djibouti. ${ }^{46}$

Dans cette requête, l'organe juridictionnel était placé devant un dilemme: accepter ou refuser de se prononcer sur une affaire de la compétence d'autres institutions. Mais en même temps il faut passer par cet organe après le refus de la demande de main levée adressée par le Procureur Général au Président et aux membres de l'Assemblée Nationale où siège "l'accusé" d'abus de pouvoir.

Le rejet des requêtes de l'opposition est devenu une tradition du conseil constitutionnel. Dans son dernier considérant, il affirme qu' "il n'entre pas dans la compétence du Conseil Constitutionnel de statuer sur les telles conclusions, qu'il suit de là que la requête susvisée est irrecevable".

En fait, la contestation contre un abus de pouvoir ou excès de pouvoir d'une administration comme un ministère ou un département d'un ministère, relève d'une autre institution : le conseil du contentieux administratif. Or celle-ci ne fonctionne pas.

Le consensus politique dans un régime démocratique repose avant tout sur le respect des actes et les décisions adoptés par une institution. Mais quel type de respect faut-il pour qu'une institution juridictionnelle fonctionne bien pour le bénéfice de tous les acteurs.es, politiques comme non politiques?

\section{Le respect formel des décisions du conseil constitutionnel}

L'institutionnalisation d'une institution politique ou d'un organe constitutionnel se manifeste par le respect d'une part de ses compétences par des organes judiciaires et politiques du pays, lesquelles sont déterminées par un cadre législatif et d'autre part l'acceptation et l'accomplissement de ses

\footnotetext{
46 Idem.
} 
décisions par les autorités judiciaires et politiques du pays. Ce sont deux conditions sine qua non pour le développement d'une juridiction constitutionnelle dans un régime pluraliste, qui se veut démocratique.

Les décisions adoptées par le conseil constitutionnel depuis 1993 n'ont pas fait l'objet de recours, ni de contestation de la part des autorités judiciaires et politiques. Le fait qu'elles relèvent de l'autorité de la chose jugée a créé un esprit de respect des décisions dudit conseil.

Cependant le respect de l'autorité de la chose jugée relève plus d'une théorie qu'une conscience nouvelle du droit constitutionnel dans le jeu politique. On peut dire qu'il y a une certaine accommodation de la part des hommes politiques à des décisions du Conseil Constitutionnel, qui ne changent pas le cours de la vie politique.

Mais toutes les décisions ne furent pas été respectées par les autorités politiques. C'est le cas de la décision n ${ }^{\circ} 96-01 / \mathrm{CC}$ des 30 et 31 juillet 1996. Le juge constitutionnel déclare d'une part que "la lettre no. 141/AN/FW du 15 juin 1996 de Monsieur le Président de l'Assemblée nationale à Monsieur le Ministre de la Justice, ne constitue pas une résolution portant autorisation de levée de l'immunité parlementaire..." ${ }^{47}$ et d'autre part que "la lettre no. 141 /AN/FW du 15 juin 1996... ne peut suppléer à l'absence d'une résolution du bureau de l'Assemblée nationale et produire les effets y attachés". ${ }^{48}$

Le respect de l'ordre institutionnel conduirait le bureau de l'Assemblée Nationale à reprendre la procédure en respectant le règlement intérieur de l'institution parlementaire, adopter une résolution et l'envoyer au Procureur de la République au lieu et place d'une lettre du président de cette dernière institution. Et la justice devrait respecter la décision du conseil constitutionnel de ne pas poursuivre la procédure judiciaire ouverte contre ces députés. Par l'attitude de l'administration judiciaire, dépendant du gouvernement (ministère), c'est l'autorité de l'institution qui est bafouée, mais aussi celle de l'institution juridictionnelle, qui n'a pas été respectée. Cette administration n'a pas remis en cause la décision du Conseil constitutionnel. Mais en poursuivant la procédure ouverte contre les accusés, elle s'appuie sur une décision, qui constate la violation d'un règlement. Reprenant les termes de R. Poussar, ${ }^{49}$ l'autorité de la chose interprétée par le conseil constitutionnel n'est qu'un mot, qui sent la poudre ou un artifice mouillé?

47 Art. 2 de la décision no. 96-01/CC des 30 et 31 juillet 1996. Le conseil constitutionnel djiboutien présente ses décisions sous forme d'article.

48 Art. 3 de la décision no. 96-01/CC des 30 et 31 juillet 1996.

49 Cité par Emmanuel Adouki, Delphine, "Contribution à l'étude de l'autorité des 


\section{CONCLUSION}

L'existence d'une justice constitutionnelle est une réalité sociopolitique en République de Djibouti théoriquement de l'indépendance du pays par emprunt de l'ex puissance coloniale. L'enjeu de son existence est de permettre l'application et le respect des principes constitutionnels contenus dans la constitution du 15 septembre 1992 par tous les acteurs.es de la vie politique et sociale. Cependant sa seule existence ne constitue pas une garantie des droits, libertés et les principes reconnus par la constitution et les traités internationaux à la personne humaine. Elle est insuffisante, c'est la culture politique et sociale et son fonctionnement qui fait d'elle une institution importante dans les nouvelles relations sociopolitiques dans ce pays.

Depuis le début de l'exercice de ses compétences en matière électorale et constitutionnelle 1993, le conseil constitutionnel s'est limité dans ses décisions par le rejet des requêtes de l'opposition sans vraiment étudier les arguments des requérants, sans utiliser les possibilités que lui offre la loi organique $n^{\circ}$ 4/AN/93/3ème L du 07 avril 1993 en ouvrant à une procédure contradictoire comme le prévoit l'art. 74 de la Loi Organique $\mathrm{n}^{\circ} 1$ / AN/92 $2^{\text {ème }} \mathrm{L}$ du 29 octobre 1992 relative aux élections. ${ }^{50}$

Dans la résolution des conflits juridiques et politiques, le conseil constitutionnel djiboutien est saisi essentiellement par les candidats de l'opposition principalement pour contester la publication des résultats des élections. Sur 15 décisions depuis 1993, il n'a jamais utilisé la procédure contradictoire alors que les arguments contenus dans certaines requêtes le conduisaient à s'appuyer sur cette procédure.

Aussi la paralysie d'autres institutions comme le Tribunal du Contentieux Administratif a joué un rôle négatif dans la résolution de certains conflits juridiques et politiques et donc du rôle de la justice dans la résolution des conflits juridico-politiques. A cela il faut ajouter le fait qu'il soit dépourvu de la compétence de prévention constitutionnelle des conflits tant juridiques que politiques pour garantir le fonctionnement des institutions et de maintenir la stabilité du système politique. Une telle prévention serait une compétence donnée au conseil constitutionnel comme la Cour constitutionnelle béninoise, qui a pu prévenir des situations explosives

décisions du juge constitutionnel en Afrique", Revue Française de Droit Constitutionnel, Paris, no. 95, juillet 2013, pp. 611.

50 Cette loi a été modifiée en 1993, ce qui a donné la Loi Organique nº2/AN/93 du 7 avril 1993. 
durant les compagnes électorales notamment pour les élections présidentielles en avisant l'imminence d'un conflit social par les propos tenus par un.e candidat.e. Si le rôle et le respect d'une juridiction constitutionnelle dépendent en grande partie des capacités de ses membres, utilisant les procédures prévues par le cadre légal, mais ils résultent aussi de l'usage des acteurs.es politiques et sociales. Or ces derniers.es, et surtout les membres de l'élite politique, ne respectent pas les décisions. Au contraire, elles/ils usent et abusent l'institution juridictionnelle à leurs bénéfices et jeux politiques. Ce qui paraît dangereux pour la stabilité politique du pays.

Dans la pratique, et en reprenant les mots de Delphine il fut un "simple ornement institutionnel du nouveau système politique". ${ }^{51}$ Les candidats. es et les partis de l'opposition saisissent le conseil constitutionnel pour résoudre un conflit juridique et/ou politique au lieu de recourir évitant ainsi la violence postélectorale comme cela se produit dans d'autres pays de l'Afrique subsaharienne. Le juge constitutionnel djiboutien n'a pas joué pleinement son rôle de gardien de la suprématie de la constitution et le respect de l'Etat de droit et des principes constitutionnels, et en particulier dans ses compétences exclusives en matière électorale.

On ne peut toutefois exclure ou sous-estimer une évolution de l'attitude du juge constitutionnel djiboutien tant pour les questions relatives aux irrégularités électorales que pour le contrôle de constitutionnalité et à la résolution des conflits juridiques et politiques, qui surviennent dans les relations sociopolitiques.

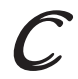

51 Emmanuel Adouki, Delphine, op. cit., p. 611.

Fecha de recepción: 18 de agosto de 2018.

Fecha de aceptación: 27 de abril de 2019. 